spécifique. Or, l'organisme est incapable de créer "de toutes pièces certaines chaînes d'amino-acides (arginine, lysine) ou de former certains noyaux cycliques (tryptophane, etc.). Cette fonction de Cyclopoièse (Osboran et Mendec) est en dehors de ses moyens de synthèse " (30). Il doit emprunter ces amino-acides aux albumines étrangères et les $\mathrm{y}$ trouver en proportions optimum. On conçoit que ce travail de reconstruction se fera avec d'autant plus d'économie que l'albumine consommée se rapproshera davantage, par la nature et le mode d'association de ces amino-acides, de l'albumine spécifique à reconstruire. La nécessité, dans le métabolisme animal, d'acides-aminés contenant un noyau aromatique ou hétérocyclique et celle de certains acides diaminés est impérieuse. Si la valeur d'une albumine est réglée par la nature et le taux de ces amino-acides, sa valeur d'utilisation est en réalité réglée par le taux du fragment, le moins abondamment représenté. Cette loi a été établie par Osborne et Mender sous le nom de "Loi du Minimum » (28).

(A suivre.)

\title{
LA TENEUR EN MATIËRE GRASSE DU BABEURRE ET LA DURÉE DU BARATTAGE PEUVENT-ELLES ÊTRE INFLUENCÉES PAR LE GENRE DE LEVAIN LACTIQUE?
}

\author{
par W. VAN DAM et B. J. HOLWERDA.
}

Cette question nous fut posée par un praticien, le Chef technique de la Fédération des fabriques coopératives des produits de laiterie de la Frise, pau de temps après le début de nos expériences concernant cette question, expériences dont les résultats sont donnés dans cet article.

Nous avons essayé 17 levains d'origines différentes ; la plupart provenaient de fabriques de produits de laiterie de la Hollande du Nord, de la Frise, de l'Ovérijssel et de la Gueldre. Nous ne pouvions, à notre grand regret, à cause de la grande distance, espérer recevoir des levains en bon état du Limbourg et du Brabant. En dehors de quatre levains provenant de Hoorn et d'un cinquième qui, environ dix ans auparavant, avait été prélevé dans une fabrique de Giekerk, les douze autres levains provenaient tous de localités différentes. 35 expériences furent faites avec ces 17 levains ; chaque jour, deux ou trois levains furent comparés avec le levain utilisé à la fabrique d'expériences. Nous étions d'avis que cette comparaison était nécessaire, car on pouvait prévoir la possibilité d'anomalies dans une ou plusieurs cultures, provoquées par l'incubation dans un lait différent de celui auquel elles étaient habituées, et la comparaison avec la culture utilisée régulièrement à Hoorn pouvait constituer un moyen de contrôle plus ou moins efficace. La méthode - pératoire fut celle des expériences décrites dans les articles précédents. 
De là crème à $20 \%$ de matière grasse, pasteurisée $\left(25\right.$ minutes à $65^{\circ} \mathrm{C}$.) fut placée une ou deux fois durant 24 heures dans la glace fondante, puis chauffée, en prenant les précautions antérieurement décrites, à la température de maturation et additionnée de levain lactique qui nous était parvenu des fabriques par la voie la plus directe. Chaque jour d'expériences, trois ou quatre comparaisons furent faites. Le barattage

Tableat $\mathbf{~}$.

\begin{tabular}{|c|c|c|c|c|c|c|c|c|c|}
\hline $\begin{array}{c}\text { No } \\
\text { crème }\end{array}$ & $\begin{array}{l}\text { Levain } \\
\text { lactique } \\
\text { addi- } \\
\text { tionné }\end{array}$ & $\begin{array}{l}\text { Tempéra- } \\
\text { ture } \\
\text { de } \\
\text { maturation }\end{array}$ & $\begin{array}{c}\text { Durée } \\
\text { de la } \\
\text { matura- } \\
\text { tion } \\
\text { heures }\end{array}$ & $\begin{array}{c}\text { Degré } \\
\text { d'acidité } \\
\text { avant } \\
\text { barat- } \\
\text { tage }\end{array}$ & $\mathrm{pH}$ & $\begin{array}{c}\text { Tours } \\
\text { par } \\
\text { minute }\end{array}$ & $\begin{array}{l}\text { Tempéra- } \\
\text { ture } \\
\text { de } \\
\text { barattage }\end{array}$ & $\begin{array}{c}\text { Durée } \\
\text { du } \\
\text { barat- } \\
\text { tage } \\
\text { minutes }\end{array}$ & $\begin{array}{c}\text { Teneur } \\
\text { en } \\
\text { matière } \\
\text { grasse } \\
\text { du ba- } \\
\text { beurre }\end{array}$ \\
\hline 1 & $7 \%$ & $12-14^{\circ} \mathrm{C}$ & $48 \mathrm{~h}$. & $89+$ & 4,46 & 253 & $15,5^{\circ} \mathrm{C}$ & 27 & $0,40 \%$ \\
\hline 2 & $7 \%$ & $12-14^{\circ} \mathrm{C}$ & $48 \mathrm{~h}$. & $90-$ & 4,45 & 253 & $15,5^{\circ} \mathrm{C}$ & $26^{\prime}$ & $0,40 \%$ \\
\hline 3 & $7 \%$ & $12-14^{\circ} \mathrm{C}$ & $49 \mathrm{~h}$. & 90 & 4,47 & 267 & $15,5^{\circ} \mathrm{C}$ & $26^{\prime}$ & $0,40 \%$ \\
\hline 4 & $7 \%$ & $12-14^{\circ} \mathrm{C}$ & $49 \mathrm{~h}$. & 90 & 4,46 & 267 & $15,5^{\circ} \mathrm{C}$ & $25^{\prime}$ & $0,40 \%$ \\
\hline
\end{tabular}

fut exécuté au laboratoire ; deux échantillons de crème furent toujours barattés simultanément dans nos petites barattes d'expériences plongées dans un réservoir-thermostat. Nous aurions bien voulu faire nos expériences à la fabrique, mais nous avons dû y renoncer à cause des difficultés que présentait l'examen d'un aussi grand nombre de levains. D'autre part, la grande "sensibilité » de nos petites barattes de laboratoire constituait un avantage. Les principaux résultats obtenus sont consignés au tableau II.

Avant ce tableau, nous donnons (tableau I) les résultats fournis par une expérience de contrôle faite avec un levain lactique connu, pour être certain qu'il y a concordance suffisante entre les résultats lorsqu'on baratte des crèmes ayant subi un même traitement préalable. Dans ce but, quatre quantités éôles de crème qui avaient été maintenues dans la glace fondante durant 24 heures, et avaient été additionnées d'une même quantité de levain lactique furent utilisées. Deux échantillons furent barattés simultanément. Ainsi que le montre le tableau I, les quatre échantillons de crème donnèrent des babeurres ayant une même teneur en matière grasse.

Les trois dernières colonnes du tableau II donnent un aperçu des résultats obtenus avec les diverses cultures. Le plus grand écart dans la teneur en matière grasse du babeurre obtenu pour une journée d'expériences était de $0,05 \%$, et nous pouvons done déjà affirmer que le genre de levains n'a pas une grande influence. Cependant, $0,05 \%$ de matière grasse est un pourcentage qui a une certaine signification. Si cet écart est plus grand que celui que l'on peut admettre comme erreur d'expé. 
Tableau II.

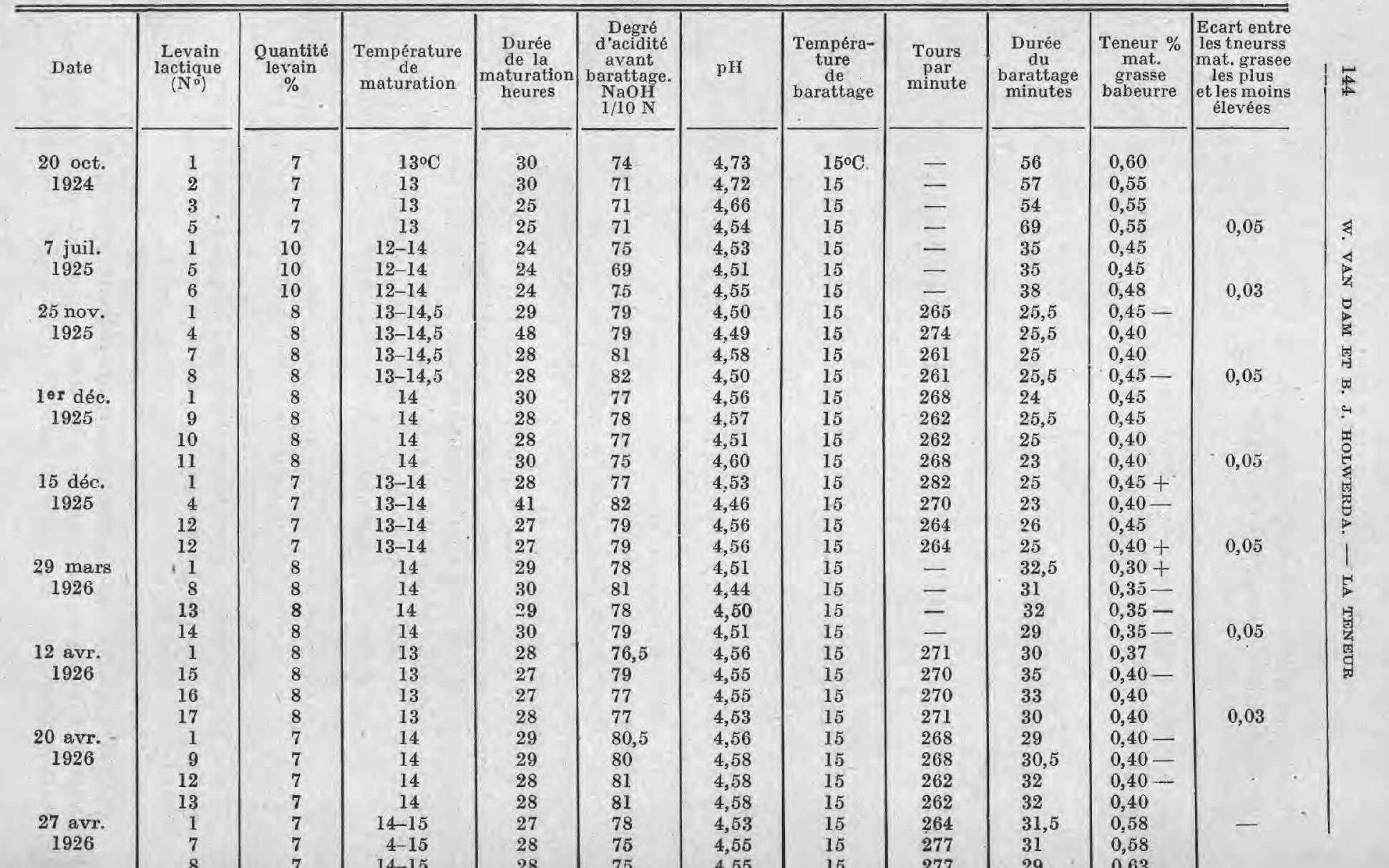


rience inévitable, inhérente à la méthode, la réponse à la question devrait être affirmative. Les données du tableau I tendraient bien à faire admettre que cette erreur est peu importante, mais les données de l'expérience du 15 décembre 1925 montrent que deux essajs faits avec le levain no 12 d'une façon absolument identique (la réfrigération, la maturation, le chauffage, etc., eurent lieu dans les mêmes conditions), donnèrent cependant une différence de $0,05 \%$, ce qui démontrerait que l'écart résultant des erreurs d'expérience inhérentes à la méthode peut atteindre cette proportion. Nous pouvons done affirmer que nos expériences n'ont pas démontré que le genre de levains lactiques peut avoir une influence sur la teneur en matière grasse du babeurre. En tout cas, s'il $y$ avait une influence, elle ne provoquerait que des écarts de la teneur en matière grasse inférieurs à $0,05 \%$.

La durée du barattage fut également peu influencée par le genre de levains lactiques. Lors de l'expérience du 20 octobre 1924, le levain 5 donna une durée de barattage notablement plus longue que les trois autres. C'était le levain très ancien provenant de Giekerk. Le motif de cette exception ne nous est pas connu. Le 7 juillet 1925, le même levain donna une durée de barattage normale. Le levain 15 donna également le 12 avril 1925 une durée de barattage présentant un écart plus grand avec celui fourni par les autreś levains que l'écart qu'on pourrait admettre comme erreur d'expérience.

Il importe de signaler, qu'en général, les levains lactiques utilisés donnèrent une bonne maturation. Seule, la maturation avec le $\mathrm{N}^{0} 4$ fut trop prolongée, mais apparemment cela n'influença pas le processus du barattage. Deux fois seulement, on a pu constater qu'il y avait dans les levains reçus un développement net de levure.

Les barattages nécessaires aux expériences décrites dans cet article et dans ceux qui l'ont précédé, ont été exécutés avec une grande précision par le technicien H. V. D. Koor.

\section{RÉsumé.}

Des expériences furent exécutées au laboratoire pour déterminer si le genre de levains lactiques additionnés à la crème exerce une influence sur la teneur en matière grasse du babeurre et sur la durée du barattage.

Les écarts constatés ne dépassent pas ceux qu'on doit considérer comme erreurs inhérentes à la méthode. Le plus grand écart constaté dans la teneur en matière grasse fut de $0,05 \%$.

Traduction : R. N. Göransson. 